\title{
PARTIAL HYSTERESIS CYCLES IN SHAPE-MEMORY ALLOYS: EXPERIMENTS AND MODELLING
}

\section{J. ORTÍN}

Departament d'Estructura i Constituents de la Matèria, Facultat de Fisica, Universitat de Barcelona, Av. Diagonal 647, 08028 Barcelona, Catalonia, Spain

\begin{abstract}
This work presents a collection of experimental pseudoelastic hysteresis cycles associated with the isothermal stress-induced martensitic transition of a $\mathrm{Cu}-\mathrm{Zn}-\mathrm{Al}$ single crystal. It is found that stress-strain trajectories retain memory of loading and unloading points, as observed for polycrystals. A particular version of the Preisach model for hysteresis is used to predict partial loops, using a limited number of experimental trajectories as input data and no adjustable parameters. Comparison between measured and predicted trajectories reveal that energy dissipation in the forward transition is different than in the reverse transition; this is confirmed by a distinct evolution with transformed fraction of the energy losses computed from two different (loading and unloading) kinds of partial cycles.
\end{abstract}

\section{1.- Introduction.}

Thermodynamic trajectories of a thermoelastic martensitic transition can be characterized by the evolution of transformed fraction or some other extensive quantity with temperature and external stress. The trajectories are widely acknowledged to display hysteresis, i.e. systematic branching every time that transformation direction is reversed [1].

Two distinctive features make the study of the hysteretic behaviour in shape-memory alloys very attractive from a fundamental point of view:

(i) Firstly, at temperature and stress rates low enough, and under well defined experimental conditions, thermodynamic trajectories are highly reproducible; the reason is that the system follows a quasistatic evolution, oriented by internal inhomogeneities, through a continuum of metastable states [2,3].

(ii) Secondly, thermodynamic trajectories depend on their previous history through the sequence of points where transformation direction is reversed, called return points $[4,5]$. This property has been observed in the thermally-induced transition of $\mathrm{Cu}-\mathrm{Zn}-\mathrm{A}$ ] single crystals [5], $\mathrm{Cu}-\mathrm{Zn} \mathrm{[6]} \mathrm{and} \mathrm{Cu}-\mathrm{Zn}-\mathrm{Al}$ [7,8] polycrystals, and in the stress-induced transition of $\mathrm{Cu}-\mathrm{Zn}-\mathrm{Al}$ [9] and $\mathrm{Ni}-\mathrm{Ti}[10]$ polycrystals. Since in all these cases the martensitic phase forms in a multiplicity of orientations, one could relate the memory of return points to a complex domain arrangement in the martensitic phase. The measurements presented here, however, show that global memory of return points is found as well in the stress-induced transition of a $\mathrm{Cu}-\mathrm{Zn}-\mathrm{Al}$ single crystal [11], a case in which all martensitic plates form with the same orientation.

In the last years there has been increasing interest in developing models capable of reproducing the particular features described [12,13]. Models of this kind may be relevant in developing a thermodynamic formulation of shape-memory alloys consistent with the fact that 
thermodynamic trajectories are multivalued [14]. These models are required as well to predict partial stress-strain-temperature trajectories, a difficulty currently hindering the practical use of shapememory alloys as regulators. In this sense the Preisach model [15] is particularly attractive: it is able to predict complicated trajectories using only a limited set of experimental data, with no adjustable parameters.

This paper is organized as follows: the Preisach model, adapted to reproduce pseudoelastic stress-strain trajectories, is introduced in section 2 , and trajectories predicted by the model are compared to experimental ones in section 3 [11]. The evolution of energy losses with transformation are analyzed in section 4 , and the conclusions of the work are given in section 5 .

\section{2.- A model for pseudoelastic stress-strain trajectories.}

In an isothermal stress-induced phase transition, the system responds to an applied uniaxial stress $\sigma$ (input) with a resulting deformation $\varepsilon$ (output). We define an elementary operator $\gamma_{\alpha \beta}$ in the way shown in figure 1. In the Preisach model [15], a superposition of these elementary operators with different $\alpha, \beta$ values $(\alpha \geq \beta)$ provides the strain $\varepsilon(t)$ corresponding to a value $\sigma(t)$ of the applied stress:

$$
\epsilon(t)=\iint_{\alpha z \beta} \mu(\alpha, \beta)\left[\gamma_{\alpha \beta} \sigma(t)\right] d \alpha d \beta
$$

The function $\mu(\alpha, \beta)$ represents the population of operators with switching values $\alpha, \beta$. Since the output of an elementary operator can only take the values 0 or 1, equation (1) reduces to:

$$
\epsilon(t)=\iint_{S^{*}(t)} \mu(\alpha, \beta) d \alpha d \beta
$$

where $S^{+}(t)$ is the region (in the triangular support $\alpha \geq \beta$ ) containing all the $(\alpha, \beta)$ points corresponding to elementary operators in the +1 state at time $t$, and only those points.
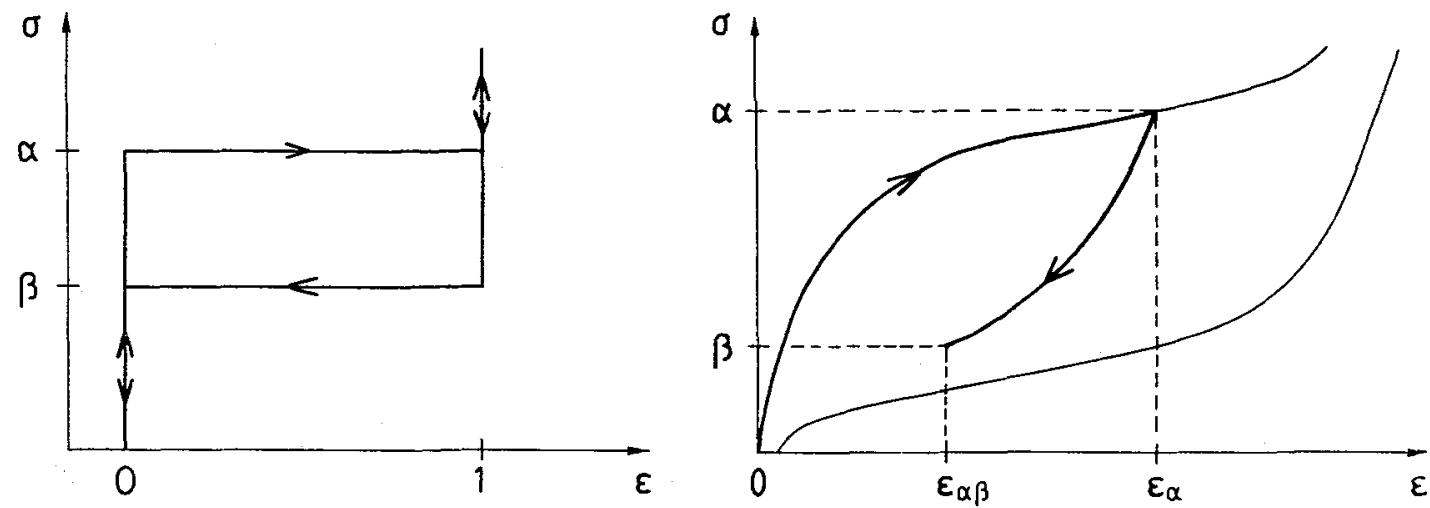

Figure 1 Left: Definition of the elementary hysteresis operator $\gamma_{\alpha \beta}$. Right: Determination of $\varepsilon_{\alpha}$ and $\varepsilon_{z \beta}$ form a first-order reversal trajectory (schematic).

It can be shown that trajectories given by equation (1) display the same global memory features than experimental tensile trajectories. Memory of the return points is stored in the particular staircase shape of $S^{+}(t)$. What makes the Preisach model particularly attractive is the possibility of 
computing the population $\mu(\alpha, \beta)$ directly from an ensemble of first-order reversal curves determined experimentally. First-order reversal trajectories are trajectories obtained from either the loading or the unloading main branches of the hysteresis cycle on reversing the direction of transformation. Figure 1 shows one of these trajectories, from which we construct the function:

$$
F(\alpha, \beta) \equiv \epsilon_{\alpha}-\epsilon_{\alpha \beta}
$$

Then, it is not difficult to show that:

$$
\mu(\alpha, \beta)=-\frac{\partial^{2} F(\alpha, \beta)}{\partial \alpha \partial \beta}
$$

A collection of experimental first-order reversal curves is plot in figure 2, together with the resulting $F(\alpha, \beta)$ function.
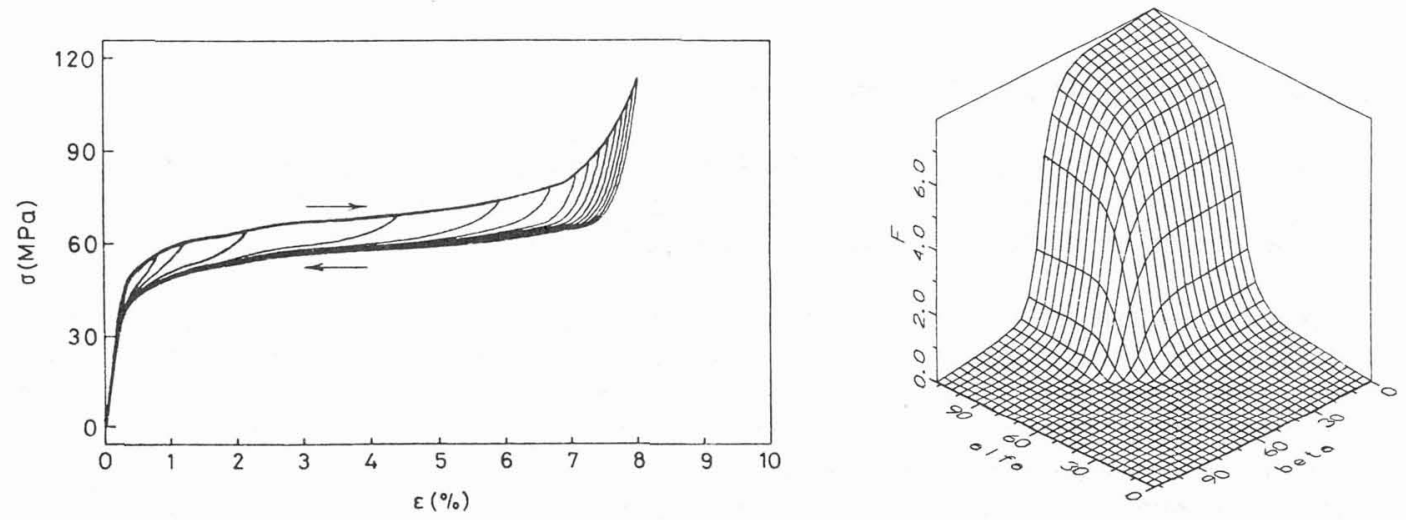

Figure 2 First-order reversal trajectories of a $\mathrm{Cu}-19.4 \mathrm{Zn}-13.1 \mathrm{Al}$ (at\%) single crystal tested in tension at room temperature, and the $F(\alpha, \beta)$ function computed from these trajectories. See reference [11] for experimental details.

\section{3.- Comparison between experimental and calculated trajectories.}

It is worth noting that equation (2) involves a double integration of the population function $\mu(\alpha, \beta)$, which on its turn is obtained as a double derivative of $F(\alpha, \beta)$. The final consequence is that predicted trajectories can be computed directly from $F(\alpha, \beta)$, avoiding the uncertainties associated with a double numeric derivative of a function affected of experimental noise. This procedure [11] has been followed to obtain the calculated trajectories of this section.

Figure 3 presents a comparison between experimental and calculated trajectories in two different cases. At left partial cycles performed from the main loading branch are considered; the agreement between model and experiment is excellent. At right we already consider partial cycles internal to other partial cycles; in this second case unloading branches are accurately reproduced but significant departures of the calculated ascending branches from the corresponding experimental ones can be observed. The disymmetry might come out from the fact that curves used to evaluate $F(\alpha, \beta)$ were unloading curves. This is an indication of possible differences in the dissipative mechanisms operative in forward and reverse transitions, consistent with measurements of acoustic emission [16], and investigated further in next section. 


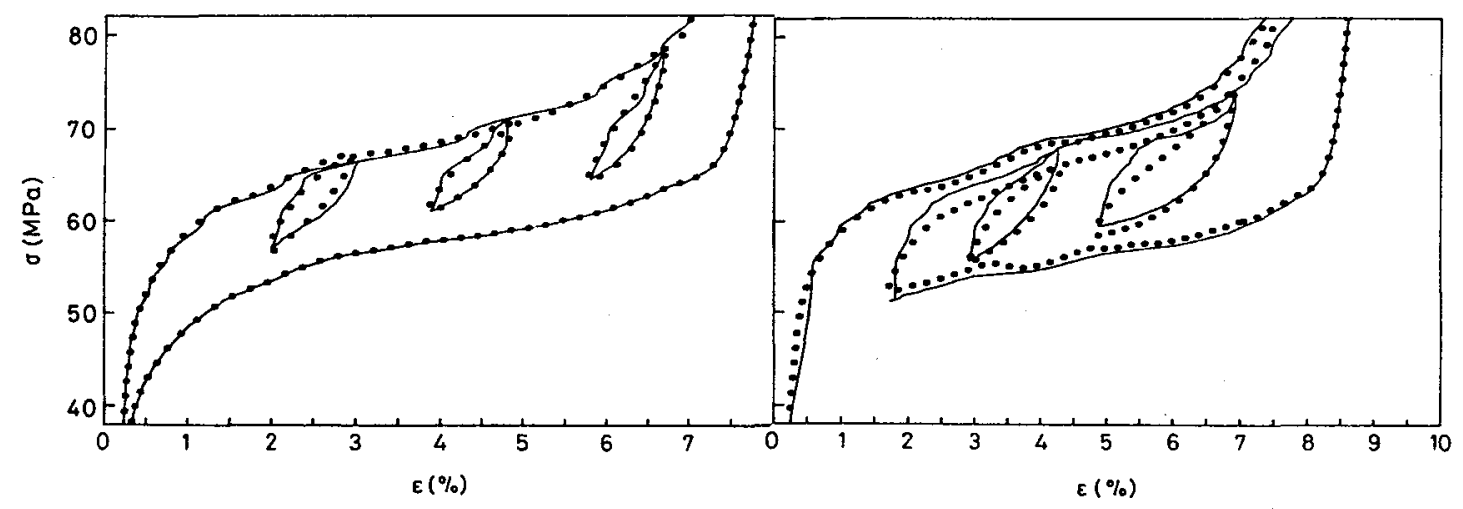

Figure 3 Comparison between experimental $(\bullet)$ and calculated (solid line) trajectories.

\section{4.- Evolution of energy losses with transformation.}

It is well known that energy losses accompanying a cyclic process can be computed as the area of the hysteresis cycle in the proper variables. As a consequence, the evolution of energy losses with transformation can be derived from the evolution of the area enclosed in consecutive first-order partial cycles [17].

It is particularly interesting to compare the results obtained with the two different kinds of first-order partial cycles available: (a) $\mathrm{P} \rightarrow \mathrm{M} \rightarrow \mathrm{P}$ cycles, formed from the main loading trajectory on unloading at different strain values (shown in figure 2), and (b) $\mathrm{M} \rightarrow \mathrm{P} \rightarrow \mathrm{M}$ cycles, obtained from the main unloading trajectory on loading again up to the maximum strain. This comparison is made in figure 4 (left). For (a), we have represented the area enclosed in the consecutive cycles, which increases sequentially with the strain value at the return point; it is worth realizing that an infinitesimal variation of the interpolated curve represents the energy consumed in an infinitesimal $\mathrm{M} \rightarrow \mathrm{P} \rightarrow \mathrm{M}$ excursion, around the forward transformation path, at the given strain. For (b), we have represented the difference in area between the complete cycle and every partial cycle; this provides a meaningful comparison with (a), since now an infinitesimal variation of the interpolated curve represents the energy consumed in an infinitesimal $\mathrm{P} \rightarrow \mathrm{M} \rightarrow \mathrm{P}$ excursion, around the reverse transformation path, at the given strain. The figure shows clearly that the two sets of values evolve very differently with transformation strain, establishing a quantitative difference between the energy dissipation processes operative in the two transition directions.

A second type of analysis using first-order partial cycles is presented in figure 4 (right). For each $P \rightarrow M \rightarrow P$ partial cycle we start at the return point and follow the unloading path, computing at each strain value the fractional area scanned in the partial cycle; the fractional area obtained varies from zero at the strain of the return point to a maximum value (the area of the partial cycle considered) at zero strain. Our results show that the evolution of energy losses with transformation is very similar, irrespective of the partial cycle considered, except at strains close to the point of transformation reversion. No significant differences can be pointed out for cycles performed along the whole loading trajectory, indicating that the dissipation mechanisms remain unaltered during the whole forward transition. Moreover, these results point out a possibility of reducing the complete set of first-order partial cycles to a unique curve, rescaling the thermodynamic variables by the values of stress and strain at the return point. Such scaling would make analytical the self-similarity observed for partial hysteresis cycles, and is currently under investigation. 

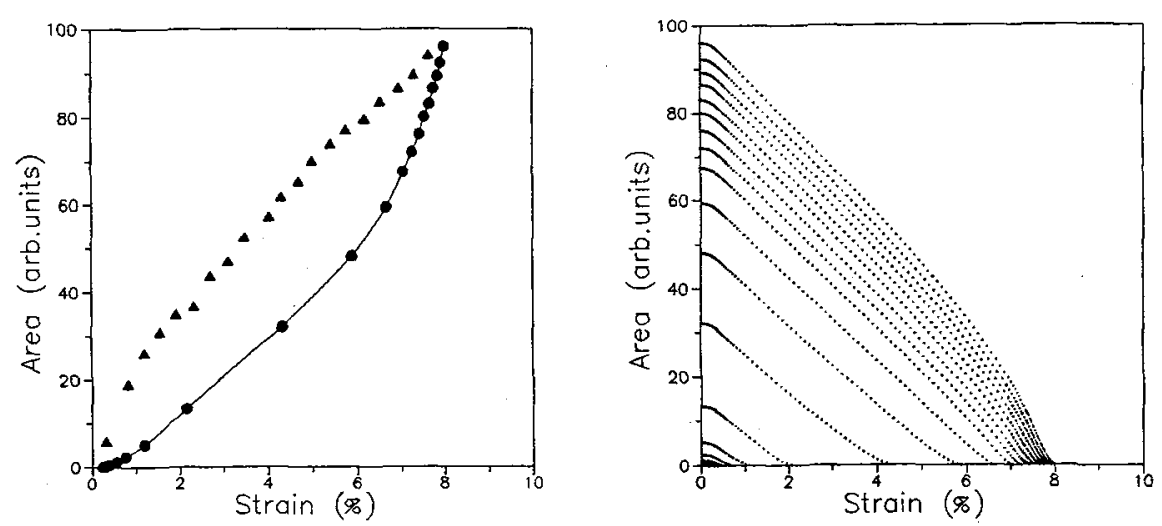

Figure 4 Left: Evolution with specimen strain of the energy dissipation, computed from the total area of consecutive first-order partial cycles. - correspond to $\mathrm{P} \rightarrow \mathrm{M} \rightarrow \mathrm{P}$ cycles, and $\Delta$ to $\mathrm{M} \rightarrow \mathrm{P} \rightarrow \mathrm{M}$ cycles. Right: Evolution with specimen strain of the fractional area of consecutive $P \rightarrow M \rightarrow P$ first-order partial cycles.

\section{5.- Conclusions.}

In thermoelastic martensitic transitions, the peculiar features of partial hysteresis cycles (particularly a global memory of points of transformation reversion) cannot be inherently related to the formation of martensitic variants with different orientations. In this work they are also found in the stressinduced transition of a single crystal, in which only one variant is formed.

These features are adequately reproduced by a Preisach model of hysteresis. The actual implementation of this model on experimental trajectories, however, reveals a dissimilar behaviour of forward and reverse transitions regarding hysteresis. This is consistent with existing results on the acoustic emission accompanying the transformation.

Distinguishing between dissipation in one and other transformation direction is not straightforward; energy dissipation measured in partial or complete closed cycles includes transformation in both directions. Nevertheless, the procedure presented in this work has revealed a distinct dissipative behaviour of the main loading and unloading trajectories, and has identified its evolution with specimen strain. Correlating such evolution with the evolution of the parent-martensite microstructure, in the two directions of transformation, would be of interest for modelling purposes

\section{Acknowledgements.}

The experimental support received from the Department of Metallurgy and Materials Engineering of the Katholieke Universiteit Leuven, in Belgium, is gratefully acknowledged. Research on martensitic transitions is supported by the CICyT under project nr. MAT89-0748. I am also acknowledged to the CIRIT (Generalitat de Catalunya) for the award of a travel grant. 


\section{References.}

[1] L Delaey, J Ortín and J Van Humbeeck, Procs. of Phase Transformations '87, G W Lorimer ed., The Institute of Metals, 1988, pp. 60-66.

[2] C J Adkins, Equilibrium Thermodynamics, Cambridge University Press, Cambridge 1983, pp. 9-10.

[3] J Ortín and A Planes, these Proceedings.

[4] L Delaey and E Aernoudt, Procs. of the International Conference on Martensitic Transformations, The Japan Institute of Metals, Nara 1986, pp. 926-933.

[5] A Planes, T Castán, J Ortín and L Delaey, J. Appl. Phys. 66, 2342 (1989).

[6] I Cornelis and C M Wayman, Scr. metall. 10, 359 (1976).

[7] W Zhongguo and Y Dazhi, Scr. metall. 22, 1245 (1988).

[8] Y I Paskal and L A Monasevich, Phys. Met. Metall. 52, 95 (1981).

[9] L Delaey, J Van Humbeeck, M Chandrasekaran, J Janssen, M Andrade and N Mwamba, Metals Forum 4, 164 (1981).

[10] J L McNichols Jr and J S Cory, J. Appl. Phys. 61, 972 (1987).

[11] J Ortín, submitted to the J. Appl. Phys.

[12] Lu Li, E Aernoudt, P Wollants, J Van Humbeeck and L Delaey, Z. Metallkde. 81, 613 (1990).

[13] I Müller and $\mathrm{H} \mathrm{Xu,} \mathrm{Acta} \mathrm{metall.} \mathrm{et} \mathrm{mater.} \mathrm{39,} 263$ (1991).

[14] J S Cory and J L McNichols Jr., J. Appl. Phys. 58, 3282 (1985).

[15] I D Mayergoyz, J. Appl. Phys. 57, 3803 (1985); Phys. Rev. Lett. 56, 1518 (1986).

[16] J Baram and M Rosen, Acta metall. 30, 655 (1982).

[17] A Planes, J L Macqueron and J Ortín, Phil. Mag. Lett. 57, 291 (1988). 